\title{
Spatially isolated palladium in porous organic polymers by direct knitting for versatile organic transformations
}

\author{
Xinbo Wang a, Shixiong Min ${ }^{\text {a,c }}$, Swapan K. Das ${ }^{\text {a }}$, Wei Fan ${ }^{\text {b }}$, Kuo-Wei Huang ${ }^{\text {, }}$, Zhiping Lai ${ }^{\text {a,* }}$ \\ a Division of Physical Science and Engineering, King Abdullah University of Science and Technology (KAUST), Thuwal 23955-6900, Saudi Arabia \\ ${ }^{\mathrm{b}}$ Chemical Engineering Department, University of Massachusetts, Amherst, MA 01003, United States \\ ${ }^{\text {c } S c h o o l ~ o f ~ C h e m i s t r y ~ a n d ~ C h e m i c a l ~ E n g i n e e r i n g, ~ B e i f a n g ~ U n i v e r s i t y ~ o f ~ N a t i o n a l i t i e s, ~ N i n g x i a ~ 750021, ~ C h i n a ~}$
}

\section{A R T I C L E I N F O}

\section{Article history:}

Received 26 May 2017

Revised 23 August 2017

Accepted 30 August 2017

\section{Keywords:}

Heterogeneous catalysis

Porous organic polymers

Direct knitting

Suzuki-Miyaura cross coupling

$\mathrm{C}-\mathrm{H}$ bond activation

\begin{abstract}
A B S T R A C T
We report here a direct knitting Method for preparation of highly robust, effective while air- and moisture-tolerant, and readily recyclable three-dimensional (3D) porous polymer-Pd network (PPPd) from the widely used $\mathrm{Pd}\left(\mathrm{PPh}_{3}\right)_{4}$. Electro-beam induced $\mathrm{Pd}$ atom crystallization was observed for the first time in organic polymer and revealed the ultrafine dispersion of palladium atoms. Challenging types of Suzuki-Miyaura couplings, reductive coupling of aryl halides and oxidative coupling of arylboronic acid were successively catalyzed by PPPd in aqueous media. Also catalytically selective $\mathrm{C}-\mathrm{H}$ functionalization reactions were achieved with orders of magnitude more efficient than conventional Pd homogeneous catalysts. The strategy developed here provides a practical method for easy-to-make yet highly efficient heterogeneous catalysis.
\end{abstract}

(c) 2017 Elsevier Inc. All rights reserved.

\section{Introduction}

Development of heterogeneous catalysts that can integrate the advantages of homogeneous catalysts has been the important target of chemists for decades yet still remains a major challenge $[1,2]$. Besides high selectivity, activity and reusability, the scalability and cost in preparation are also factors that must be considered for practical usage. Although progresses in surface organometallic chemistry $[3,4]$, zeolitic supports of so-called "ship-in-bottle" catalyst [5], high surface area material such as metal-organic frameworks (MOFs) supported catalyst [6-8], single-atom catalyst (SAC) based on atomically dispersed metal atoms on the surfaces of metal crystal or graphene [9-11], have been well documented and made an essential contribution to the progress of research in heterogeneous catalysis [12-14], these methods are costly and not readily scalable, thus do not meet industrial demands. Single atom of palladium anchoring to a relatively cheaper carbon nitride $\left(\mathrm{C}_{3} \mathrm{~N}_{4}\right)$ has been achieved recently by stepwise loading metal-salt and a subsequent reduction [15], but introducing other coordination ligands, which may fully determine the properties of the catalyst, is still challenging.

Whereas supported noble metal heterogeneous catalysis has led to a plethora of effective catalysts used in practical applications,

\footnotetext{
* Corresponding author.

E-mail address: zhiping.lai@kaust.edu.sa (Z. Lai).
}

porous organic polymers (POPs) are arguably one of the most ideal supports with unique features including excellent chemical tunability, insolubility but with adjustable wettability, recyclability, and so on [16-18]. Many organometallic and transition metal coordination catalysts contain electron-rich arene substituent groups, which from the structural view point are able crosslinked to form POP frameworks. While at the same time, their soluble nature allows easy purification from aggregated clusters and other byproducts, which is unachievable when reduction occurs inside the insoluble network. A series of heterogeneous catalysts supported on POPs have been reported and excellent selectivity and higher activity than the corresponding homogeneous counterparts were observed $[19,20]$. By crosslinking benzene and triphenylphosphine followed by dispersing $\mathrm{PdCl}_{2}$ into the framework, a highly active and stable Pd (II) organometallic heterogeneous catalyst was obtained by Tan et al. [21,22]. However, these catalysts are prepared by the conventional step-by-step method as shown in the top part of Fig. 1, where metal salts are loaded into the POP structure. In Tan's method, to obtain Pd in zero valence, a further reduction step is required. All of these post treatments will inevitable cause metal aggregation and result in decrease of reactivity and selectivity.

In this study, we utilized tetrakis(triphenyl phosphine) palladium $\left(\mathrm{Pd}\left(\mathrm{PPh}_{3}\right)_{4}\right)$ as precursor, and for the first time in one step successfully obtained a POP structure with nearly single-atom dispersed $\mathrm{Pd}$, through crosslinking the four triphenylphosphine 


\section{Step-by-Step Approcach (Widely-used)}

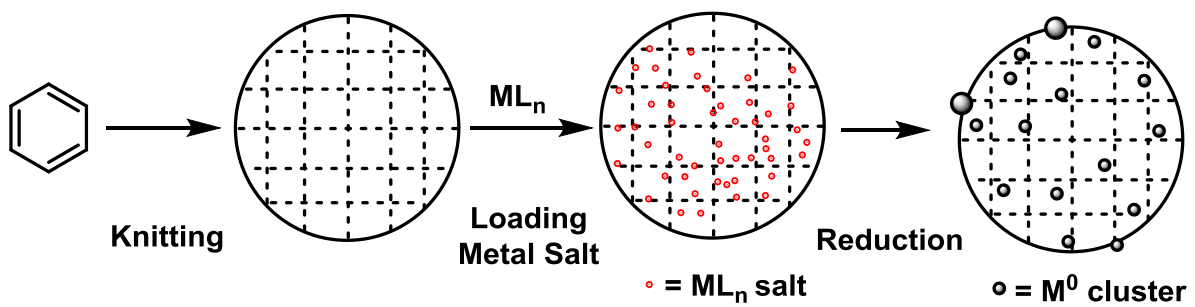

\section{Direct Knitting Approach (This work)}

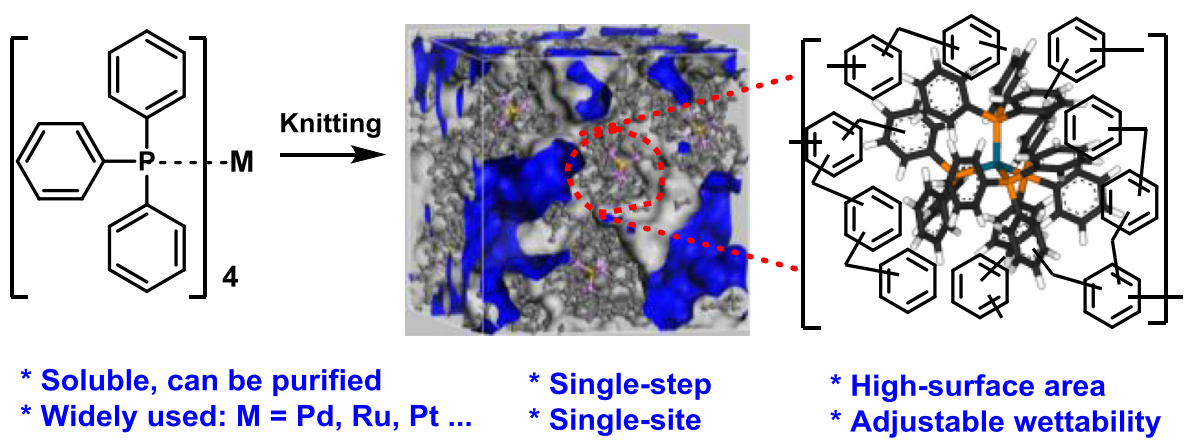

Fig. 1. Illustration of the difference between the conventional step-by-step approach and the direct knitting method.

ligands of $\mathrm{Pd}\left(\mathrm{PPh}_{3}\right)_{4}$, as illustrated in the bottom part of Fig. 1. Pd $\left(\mathrm{PPh}_{3}\right)_{4}$ is one of the most widely used homogeneous coordination catalysts but suffering from air sensitivity and poor recyclability. While the crosslinked catalyst, which was named as PPPd, showed not only higher catalytic efficiency that surpassed its homogeneous form, but also much better stability and recyclability that allowed the catalytic reactions to be carried out in aqueous solutions under open conditions.

\section{Experimental section}

\subsection{Catalyst PPPd synthesis}

Benzene (Aldrich, $0.8 \mathrm{~g}, 10 \mathrm{mmol}$ ), dimethoxymethane (Aldrich, $2.3 \mathrm{~g}, 30 \mathrm{mmol}$ ) and $\mathrm{Pd}\left(\mathrm{PPh}_{3}\right)_{4}$ (Aldrich, $0.6 \mathrm{~g}, 0.5 \mathrm{mmol}$ ) were mixed together in 1,2-dicholoroethane (Aldrich, $20 \mathrm{ml}$ ). Protected with nitrogen and cooled with ice, anhydrous $\mathrm{FeCl}_{3}$ (Aldrich, $4.8 \mathrm{~g}, 30 \mathrm{mmol}$ ) was added portion by portion. The reaction mixture was then moved to oil bath and heated to $80^{\circ} \mathrm{C}$ for $24 \mathrm{~h}$. After cooling down to r.t., the resulted precipitate was immersed and washed with methanol until the filtrate was clear. Khaki solid powder PPPd was obtained after drying in vacuum oven at $70^{\circ} \mathrm{C}$ for $48 \mathrm{~h}$.

With similar procedure, $50 \mathrm{~g}$ PPPd was produced in a scale-up experiment.

\subsection{General procedure for Suzuki-Miyaura cross coupling reaction [22]}

Inside an argon atmosphere glove box, halide $(0.5 \mathrm{mmol})$, boronic acid $(0.75 \mathrm{mmol}), \mathrm{K}_{3} \mathrm{PO}_{4}(1.5 \mathrm{mmol})$, PPPd (4 mg, $1.4 \mathrm{wt} \% \mathrm{Pd}$, $0.0005 \mathrm{mmol})$, solvent $\left(2 \mathrm{ml}, \mathrm{H}_{2} \mathrm{O} / \mathrm{EtOH} \mathrm{v} / \mathrm{v}=2: 3\right)$ were mixed in a $5 \mathrm{ml}$ vessel and sealed. Then it was moved out of the glove box and heated in an oil bath to $80^{\circ} \mathrm{C}$ for 2-6 h. After cooled down to r.t. for about half an hour, the mixture was filtered. Take a small amount of the liquid for GC analysis. The solid part was washed three times with ethanol. The combined liquid was dried and purified by column to give pure coupling product. For recycle experiments, the solid part after washing was degassed and used into the next cycle. Combing the recycled catalyst from $\mathrm{X}$ reaction times was marked as Re-PPPd-X.

\subsection{General procedure for Ullmann reductive homo-coupling reaction}

Aryl halide (0.5 mmol), $\mathrm{K}_{3} \mathrm{PO}_{4}(1.5 \mathrm{mmol}), \mathrm{PPPd}(8 \mathrm{mg} * 1.4 \mathrm{wt} \%$ $\mathrm{Pd}, 0.001 \mathrm{mmol})$, solvent $\left(2 \mathrm{ml}, \mathrm{H}_{2} \mathrm{O} / \mathrm{EtOH}^{\mathrm{v}} / \mathrm{v}=2: 3\right)$ were mixed in a $5 \mathrm{ml}$ vessel and sealed. Then it was heated in an oil bath at $80^{\circ} \mathrm{C}$ for $20 \mathrm{~h}$. After cooled down to r.t., the mixture was filtered. Take a small amount of the liquid for GC analysis. The solid part was washed three times with ethanol. The combined liquid was dried and purified by column to give pure coupling product.

\subsection{General procedure for aryl boronic acid oxidative homo-coupling reaction}

Aryl boronic acid $(0.5 \mathrm{mmol}), \quad \mathrm{K}_{3} \mathrm{PO}_{4} \quad(1.5 \mathrm{mmol}), \quad \mathrm{PPPd}$ (4 mg*1.4 wt\% Pd, $0.0005 \mathrm{mmol})$, solvent $\left(2 \mathrm{ml}, \mathrm{H}_{2} \mathrm{O} /\right.$ EtOH $\mathrm{v} /$ $\mathrm{v}=2: 3$ ) were mixed in a $5 \mathrm{ml}$ vessel and sealed. Then it was heated in an oil bath at $80^{\circ} \mathrm{C}$ for $6 \mathrm{~h}$. After cooled down to r.t. for about half an hour, the mixture was filtered. Take a small amount of the liquid for GC analysis. The solid part was washed three times with ethanol. The combined liquid was dried and purified by column to give pure coupling product.

2.5. General procedure for direct $\mathrm{C}-\mathrm{H}$ bond selective alkoxylation and halogenation reactions [23]

Benzoquinoline $(0.5 \mathrm{mmol}), \mathrm{PhI}(\mathrm{OAc})_{2} \quad(1.0 \mathrm{mmol})$, solvent $(4 \mathrm{ml})$ and PPPd $(15 \mathrm{mg} * 1.4 \mathrm{wt} \% \mathrm{Pd}, 0.002 \mathrm{mmol})$ were mixed together in a $10 \mathrm{ml}$ glass vessel under air. Then it was sealed and heated in an oil bath. The reaction was monitored by GC-MS.

\subsection{Intermolecular regioselective $\mathrm{C}-\mathrm{H}$ alkenylation of indole [24]}

Indole $(0.5 \mathrm{mmol})$, copper(ii) acetate $(0.8 \mathrm{mmol})$, alkene ( $1.5 \mathrm{mmol})$ and PPPd ( $8 \mathrm{mg} * 1.4 \mathrm{wt} \% \mathrm{Pd}, 0.001 \mathrm{mmol})$ were mixed together in a $10 \mathrm{ml}$ glass vessel under air, then DMF/ DMSO $(9: 1 \mathrm{v} / \mathrm{v}, 4 \mathrm{ml})$, was added and the vessel was sealed to heat by 
oil bath at $80^{\circ} \mathrm{C}$ for $12 \mathrm{~h}$. After cooled down to r.t. for about half an hour, the mixture was filtered. Take a small amount of the liquid for GC analysis.

\subsection{Material characterization}

${ }^{13} \mathrm{C}$ and ${ }^{31} \mathrm{P} \mathrm{CP} / \mathrm{MAS}$ measurements were performed using a Bruker $400 \mathrm{M}$ MAS system with Adamantane and $\mathrm{NH}_{4} \mathrm{H}_{2} \mathrm{PO}_{4}$ as internal reference for $\mathrm{C}$ and $\mathrm{P}$ respectively. Liquid $\mathrm{NMR}$ data were recorded on Bruker $600 \mathrm{M}$ (for ${ }^{1} \mathrm{H}$ ) and $150 \mathrm{M}$ (for ${ }^{13} \mathrm{C}$ ) with $\mathrm{CDCl}_{3}$ or $\mathrm{D}_{6}$-DMSO as solvent. Fourier transform infrared spectroscopy (FTIR) was performed on a Nicolet iS10 Smart FTIR spectrometer with Smart OMNI Transmission ranging from $4000 \mathrm{~cm}^{-1}$ to $400 \mathrm{~cm}^{-1}$, Thermo Scientific, USA. CHN elemental analysis was carried out in a Flash 2000 organic elemental CHN analyzer, Thermo Scientific, USA. Thermogravimetric analysis (TGA) and differential thermal analysis (DTA) of samples were carried out in a TGA instruments thermal analyzer Netzsch leading thermal analyzer TG 209, under $\mathrm{N}_{2}$ flow of rate $20 \mathrm{ml} / \mathrm{min}$. Scanning electron microscope images (SEM) were obtained on Quant 200 SEM operated at an accelerating voltage of $5 \mathrm{keV}$, spot size 3, SEM-EDS was performed on the same SEM equipment with an accelerating voltage of $10 \mathrm{keV}$, spot size 6 . Transmission electron microscopic images
(TEM) of the samples were recorded on a high resolution electron microscope Titan ST TEM at $300 \mathrm{kV}$. Nitrogen physisorption isotherms were measured using a Micromeritics ASAP 2420 automatic volumetric adsorption apparatus, at $77 \mathrm{~K}$. The samples were degassed at $393 \mathrm{~K}$ for $48 \mathrm{~h}$ under high vacuum prior to the gas adsorption measurements.

\section{Results and Discussion}

$\left(\mathrm{Pd}\left(\mathrm{PPh}_{3}\right)_{4}\right)$ was directly knitted into a POP structure, termed as PPPd, using the hyper-crosslinking method (experimental section).[21] FTIR spectroscopy of PPPd (Fig. 2a) and ${ }^{13} \mathrm{C} \mathrm{CP} / \mathrm{MAS}$ NMR spectrum (Fig. 2b) displays a similar bone structure to KAPs $\left(\mathrm{Ph}_{-} \mathrm{PPh}_{3}\right)$-Pd reported by Tao et al.[22] Specifically, absorption peaks (Fig. 2a) around 3040, 2000-1600, 1250-1000, and 900$690 \mathrm{~cm}^{-1}$ present the aromatic $\mathrm{C}-\mathrm{H}$ bonds in stretching mode, overtones region, in-plane blending, and "opp" bands respectively. The peak at $2927 \mathrm{~cm}^{-1}$ can be attributed to the alkyl C-H stretch vibration, indicating the existence of methylene linker formed from dimethoxymethane. The existence of $\mathrm{P}-\mathrm{Ar}$ was evidenced by the P-Ar stretching $1130-1090 \mathrm{~cm}^{-1}$ medium in IR. The ${ }^{13} \mathrm{C} \mathrm{CP} /$ MAS NMR spectrum revealed that PPPd exhibits two resonance peaks at 136 and $127 \mathrm{ppm}$ which can be attributed to the substi-
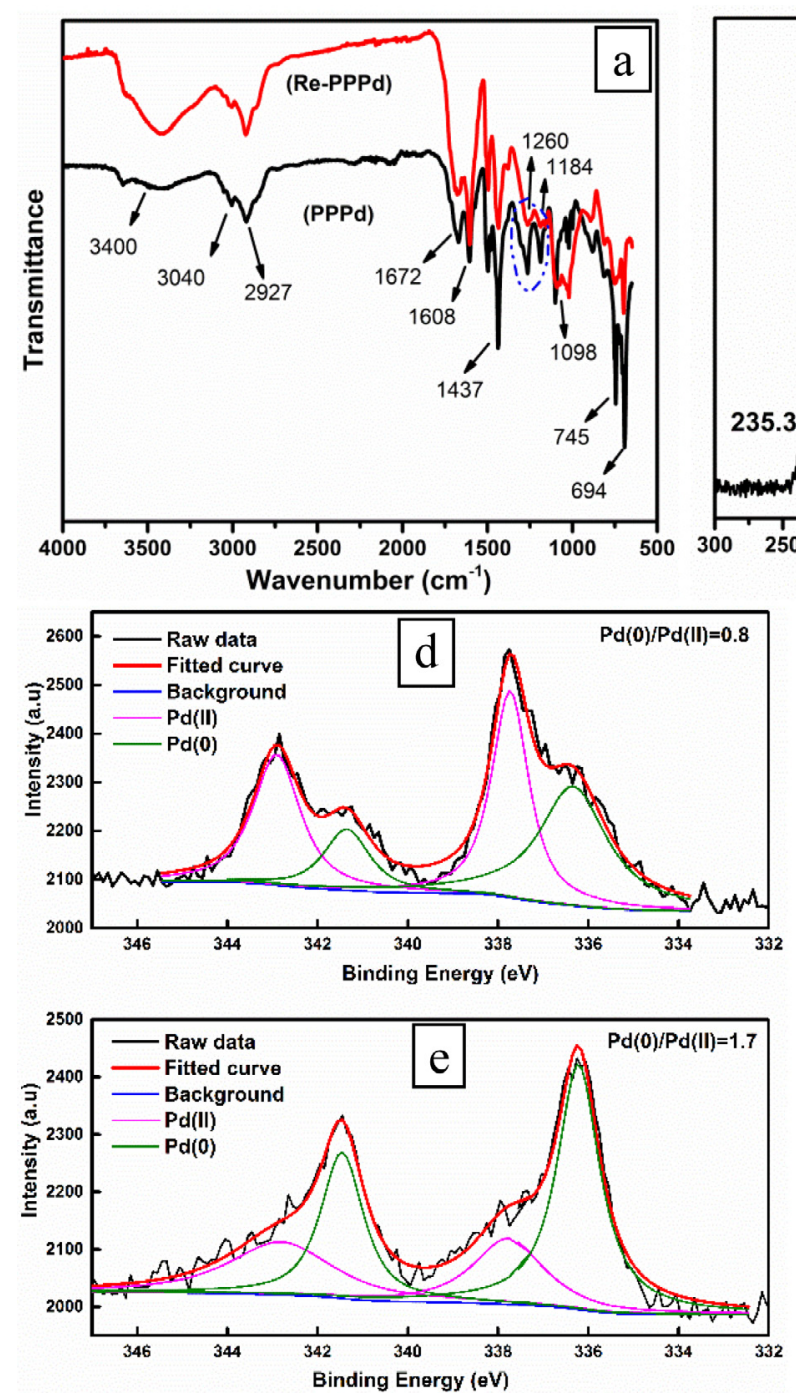
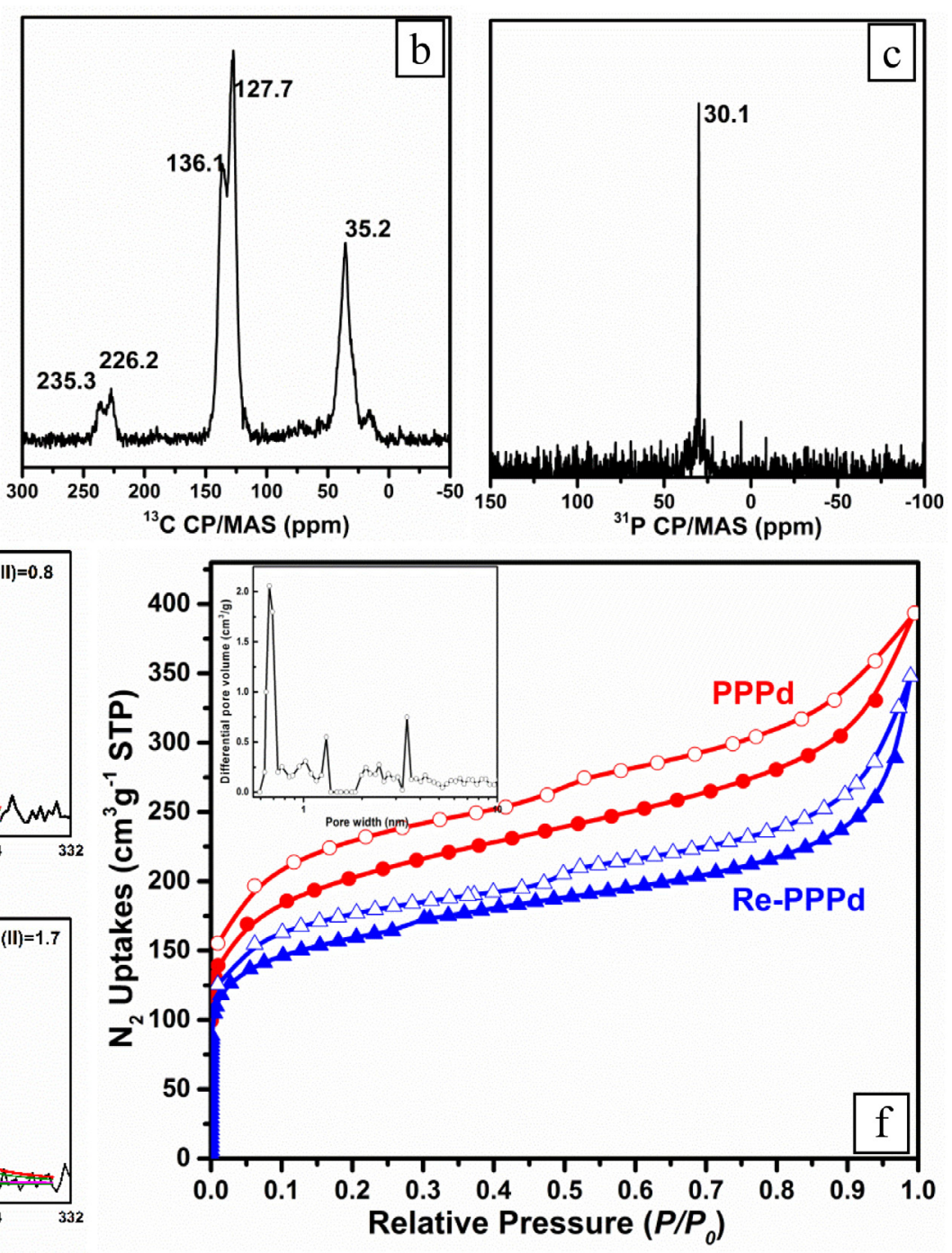

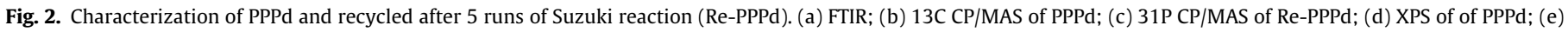
XPS of Re-PPPd; (f) Nitrogen adsorption isotherms at $77 \mathrm{~K}$, insert pore width distribution based on NLDFT analysis of the adsorption isotherms. 
tuted and non-substituted aromatic carbon respectively, and one peak at $35 \mathrm{ppm}$ belonging to the methylene linker carbon.[22] However, the new bands at 1260 and $1184 \mathrm{~cm}^{-1}$ in the FTIR spectra (Fig. 2a) as well as the chemical shift at $30 \mathrm{ppm}$ in ${ }^{31} \mathrm{P} \mathrm{CP} / \mathrm{MAS}$ NMR (Fig. 2c) both suggest that $\left(\mathrm{Pd}\left(\mathrm{PPh}_{3}\right)_{4}\right)$ were oxidized to form $\mathrm{P}=\mathrm{O}$ bonds in the POP structure. Elemental analysis (EA) measured by inductively coupled plasma atomic emission spectroscopy (ICPAES) reveals a high Pd loading of $1.4 \mathrm{wt} \%$ in PPPd, and trace amount of Fe left inside the material (200 ppm). The XPS spectra (Fig. $2 \mathrm{c}$ ) reveals mixed oxidation states $\left(\mathrm{Pd}^{0}\right.$ and $\left.\mathrm{Pd}^{\mathrm{II}}\right)$ for $\mathrm{Pd}$ species, which is due to the partial oxidation of $\left(\mathrm{Pd}\left(\mathrm{PPh}_{3}\right)_{4}\right)$ that is consistent to the FTIR and NMR results. Such a mixed-valence catalyst is beneficial in carrying out versatile transformation reactions compared to single valence catalysts [25-28].

Nitrogen adsorption measurement were conducted to analyze the porous properties of PPPd. As shown in Fig. $2 \mathrm{f}$, the isotherms displayed a typical type I curve with a steep increase of nitrogen gas uptake at a low relative pressure $\left(\mathrm{P} / \mathrm{P}_{0}<0.01\right)$, indicating the abundant presence of permanent microporosity [6]. Moreover, the slight hysteresis loop with gradual increasing gas uptake reflects the presence of mesoporosity, while a second steep increase at a high relative pressure $\left(\mathrm{P} / \mathrm{P}_{0}=0.9-1\right)$ could be attributed to the macropores. Consistently, the pore size distribution analysis of PPPd as shown in Fig. $2 \mathrm{f}$ (insert) gave two main pore size distributions of 0.7 and $3.4 \mathrm{~nm}$ corresponding to the micro and mesopores, respectively. Such a structure with mixed-sized pores has been found to be beneficial for anchoring metal catalysts by accelerating the mass transport of reactants and products $[22,29]$. The BET surface area and pore volume of the assynthesized PPPd were determined to be $702 \mathrm{~m}^{2} / \mathrm{g}$ and $0.546 \mathrm{~cm}^{3} / \mathrm{g}$, respectively. As demonstrated below, this novel porous 3D architecture offers more accessible active sites and facilitate mass transport to achieve efficient catalysis [29,30].

Fig. 3a, b show scanning electron microscopy (SEM) images of as-synthesized PPPd, in which a $10-\mu \mathrm{m}$ 3D nanoflower-like structure was constructed from interconnected and slightly bended nanosheets with thickness of ca. $80 \mathrm{~nm}$. The macropores between nanosheets are observable. The transmission electron microscopy (TEM) image (Fig. 3c) shows that most of the nanosheets have dimensions of several microns and an average thickness of about
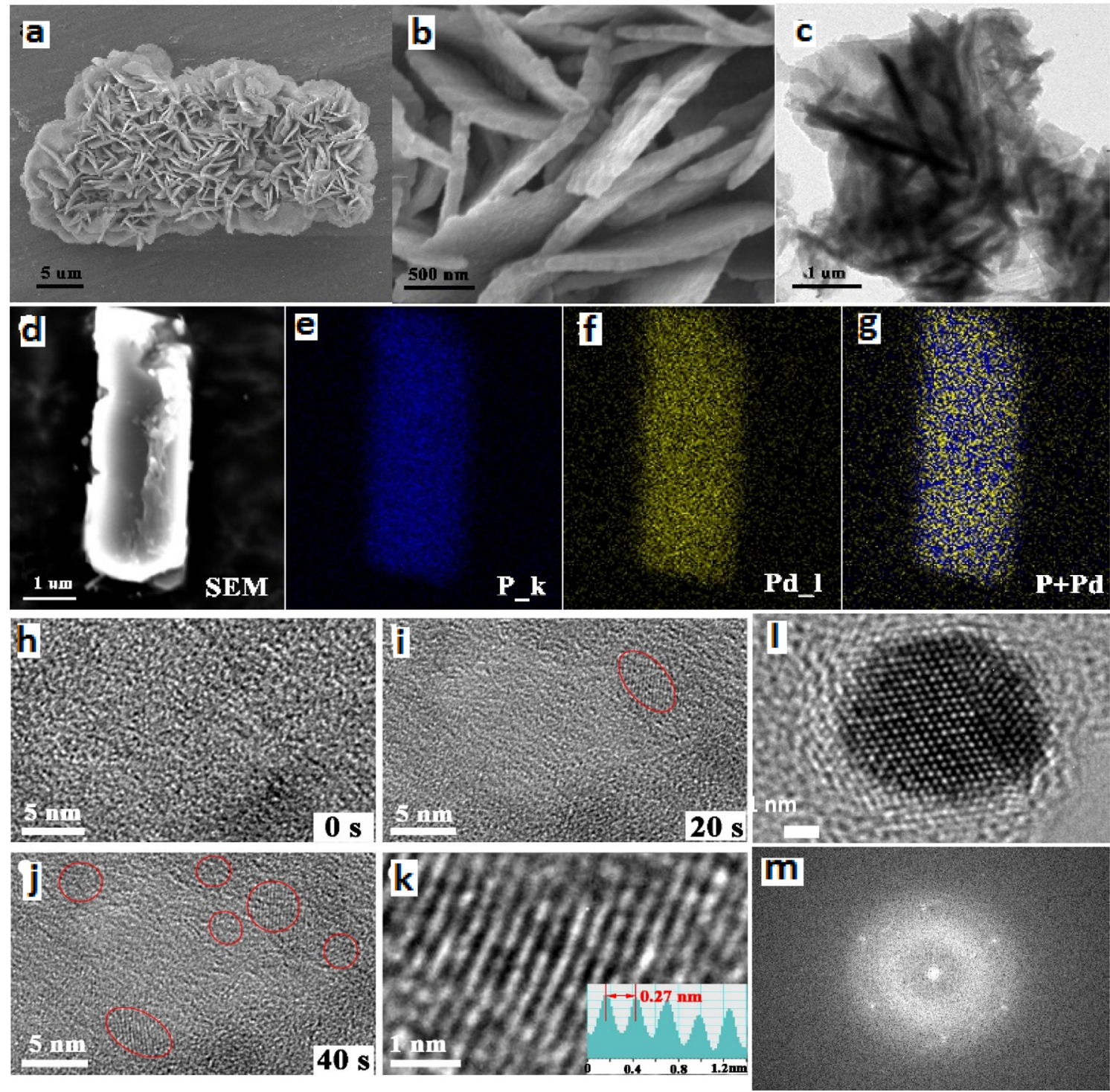

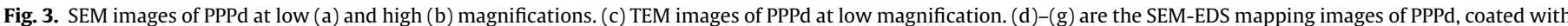

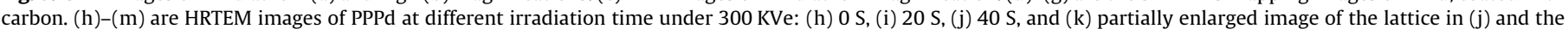
insert image in (k) shows the lattice distance which matches Pd lattice. (l) a crystalline particle found after $1 \mathrm{~h}$ irradiation together with its FFT (m). 
$100 \mathrm{~nm}$, which are consistent with SEM results. This type of assembly architecture allows for easy access to the available high surface area thus benefiting catalysis, while the macropores between each flake together with the micropores inside the nanosheet will benefit mass transport [29,30]. SEM-EDS elemental mappings (Fig. 3dg) clearly indicate that the $\mathrm{Pd}\left(\mathrm{PPh}_{3}\right)_{4}$ moiety is uniformly dispersed throughout the framework. An interesting electro-beam irradiation induced metal atoms aggregation-crystallization was observed under HRTEM imaging process. As shown in Fig. $3 \mathrm{~h}-\mathrm{m}$, no metal agglomerates could be observed at the beginning of imaging $(0 \mathrm{~s})$, while an obscure crystalline lattice corresponding to $\mathrm{Pd}$ (1 111 ) plane was developed after $20 \mathrm{~s}$, and eventually $3-5 \mathrm{~nm}$ crystalline Pd domains are observable due to the fusion of spatially isolated Pd atoms. It is known that organic polymers could be burned away under TEM beam, when the surface polymer was burned out by the eletro-beem, the metal atoms were able to assemble and crystallize. Such a time-dependent nanoparticle formation process further revealed that the palladium atoms are spatially isolated in the framework of PPPd. To the best of our knowledge, this is the first direct observation of Pd crystalline nanoparticle formation induced by electro-beam irradiation on a polymer support.

The Suzuki-Miyaura coupling reactions of Aryl iodide and aryl boronic acid were first chosen as standards to test the reactivity and recyclability of the PPPd. All of the substrates, involving trifluoromethyl, pyridine, furane, ferrocene functionalized or bisboronic acid, gave almost quantitative yield within $1 \mathrm{~h}$ in an aqueous medium (Table 1). Moreover, the catalyst can be easily recov- ered by simple filtration, and no obvious loss in the catalytic efficiency was observed after 10 repeated cycles (Fig. 4). To distinguish whether supported heterogeneous or dissolved homogeneous Pd species were the real responsible catalysts, "hot filtration" tests were adopted. Here, the reaction of chlorobenzene and phenylboronic acid (Table 2, entry 12) was stopped after $1 \mathrm{~h}$, the solid was filtered out from the hot solution and the mother filtrate was allowed to react for another $5 \mathrm{~h}$ under the similar conditions. GC analysis of the mixture showed no significant progress in the reaction occurred. Meanwhile, elemental analysis of the filtrate after the first cycle shown Pd content less than $50 \mathrm{ppb}$, indicating that no appreciable amount of Pd dissolved, which could account for the preservation of catalytic activity. Characterization of the recycled catalyst after 5 runs (Re-PPPd) produced identical spectrums as the fresh catalyst (Fig. 2). The BET surface area and the pore volume slightly decreased to $657 \mathrm{~m}^{2} / \mathrm{g}$ and $0.538 \mathrm{~cm}^{3} / \mathrm{g}$ in Re-PPPd, respectively. This was mainly due to the loss of microporosity (Fig. 2f), which could possibly be occupied by some impurities. It should be emphasized that unlike phosphine-coordinated homogeneous organometallic catalysts that are commonly unstable in air, no observable change in the morphology or the catalytic efficiency of the PPPd was found after exposure to air for several months. The enhancement of catalyst's stability is also observed in other porous material supported initially low-stable catalyst systems [31,32], most likely benefited from the spatial isolation effect of the framework by inhibiting aggregation.

The less active coupling partners for Suzuki-Miyaura reactions were then also investigated. First, the multifunctionalized aryl bro-

Table 1

PPPd-catalyzed Suzuki-Miyaura coupling reactions of Aryl iodide and aryl boronic acid.

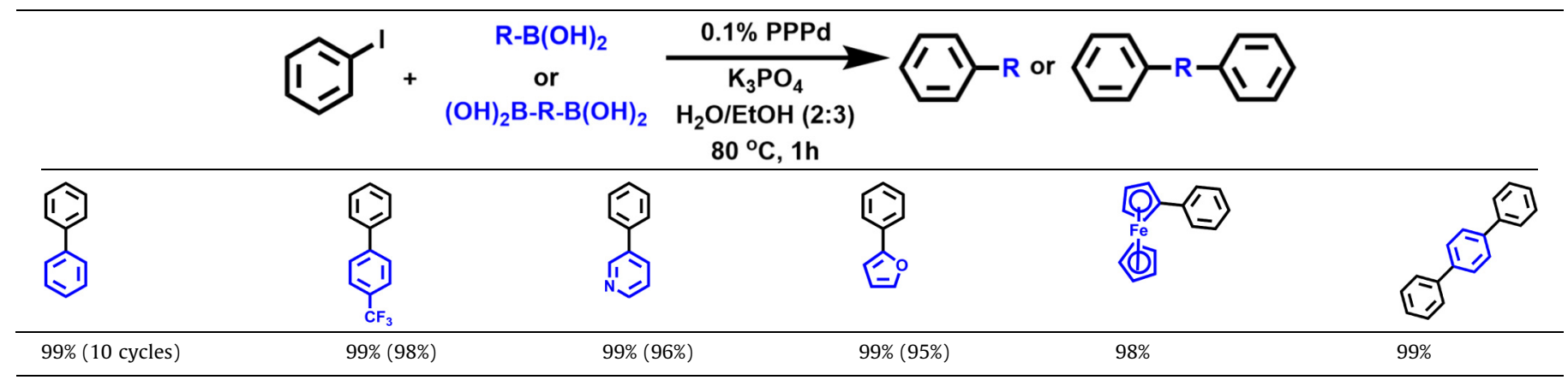

GC yield, isolated yield in parentheses.

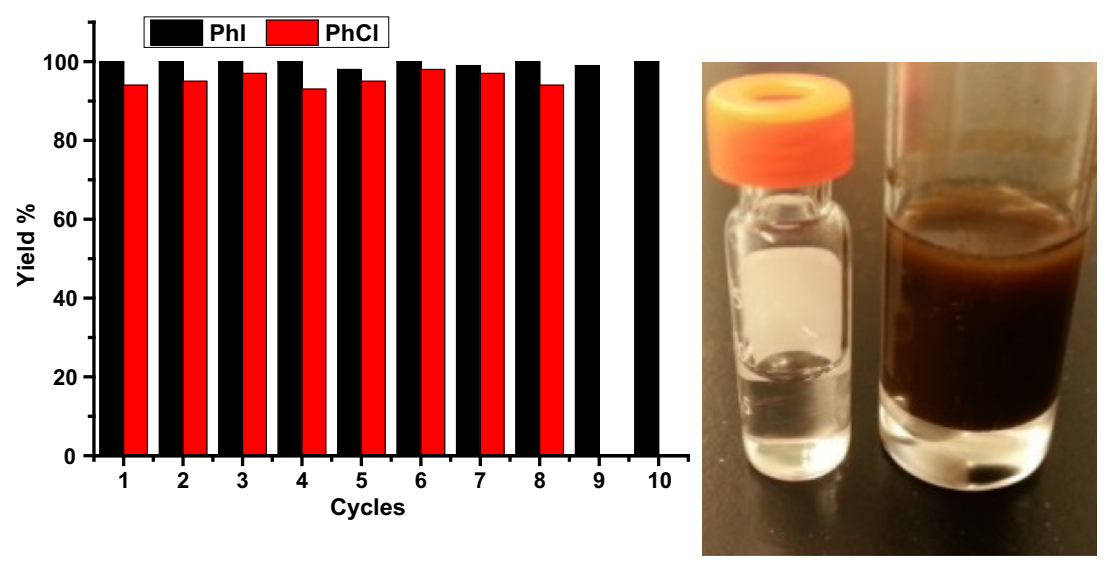

Fig. 4. Reusability test of PPPd in Suzuki-Miyaura coupling reactions (left) and a typical reaction mixture as well as the liquid phase after filtration (right). 
Table 2

PPPd-catalyzed Suzuki-Miyaura coupling reactions of challenging partners.

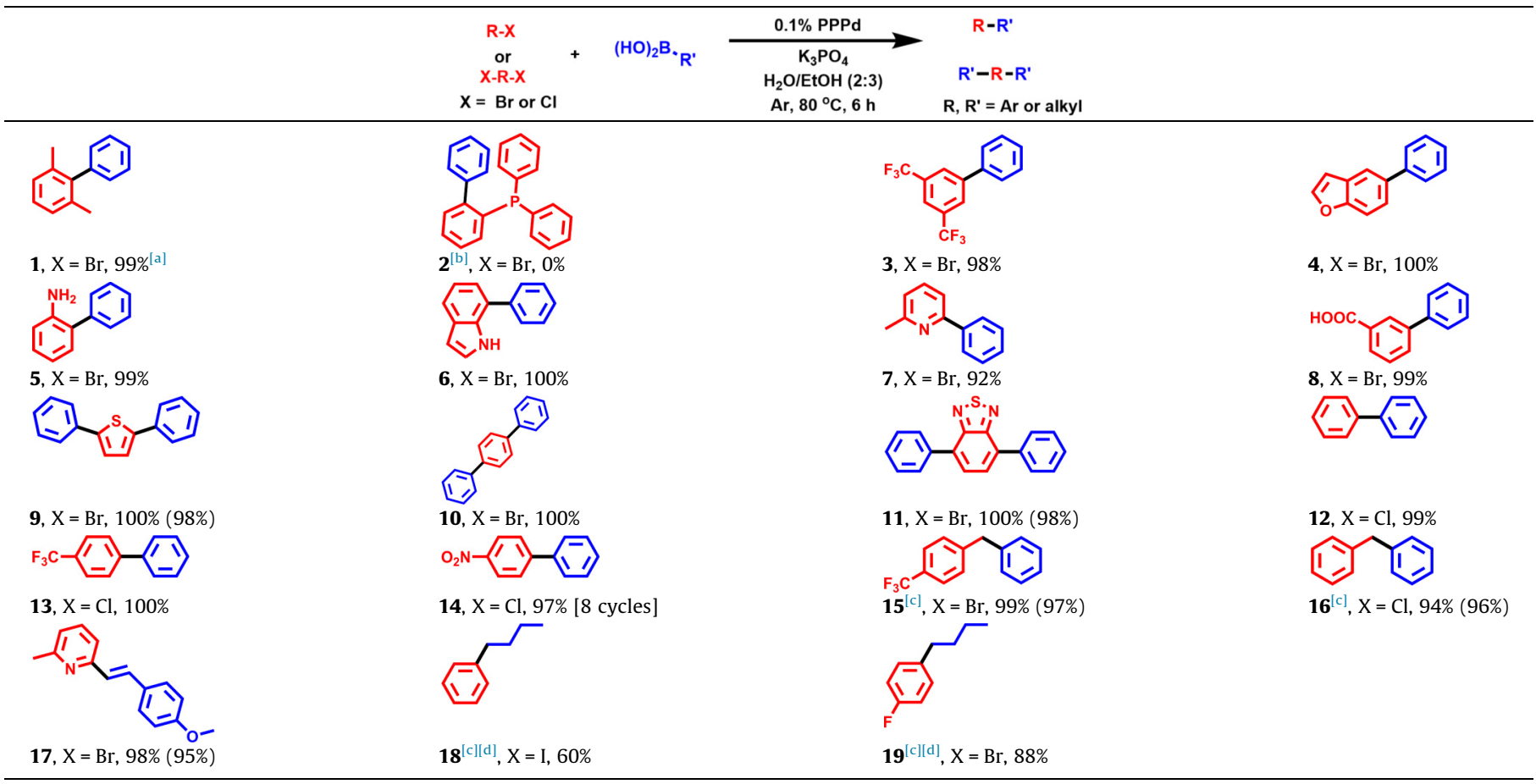

[a] GC yield with isolated yield in parentheses.

[b] $1 \mathrm{~mol} \%$ Pd was used.

[c] $0.2 \mathrm{~mol} \%$ Pd was used.

[d] $24 \mathrm{~h}$.

mides were employed which again displayed encouraging reactivity and selectivity (Table 2, entry 1 and 3-11). It should be mentioned that substrates employed here with functions of fluoride, furan, amine, indole, pyridine, carboxylic acid, and thiophene, are all general and important intermediates for the synthesis of agrochemicals, pharmaceuticals and natural products. The bipolar benzo[1,2,5]thiadiazole has wide applications in organic lightemitting diodes (OLEDs) as an emissive group [33,34]. The excellent activity and functional group tolerance of PPPd revealed its high potential in real applications in these fields. No coupling product was obtained when (2-bromophenyl) diphenylphosphane was employed as substrate, possibly due to its sterically hindered nature, suggesting that the reactions occur inside the pores. The activation of the $\mathrm{C}-\mathrm{Cl}$ bond $(\sim 79 \mathrm{kcal} / \mathrm{mol})$ is much more difficult than the $\mathrm{C}-\mathrm{Br}(\sim 66 \mathrm{kcal} / \mathrm{mol})$ and $\mathrm{C}-\mathrm{I}(\sim 52 \mathrm{kcal} / \mathrm{mol})$ bonds due to its slow oxidative addition to palladium, especially in heterogeneous catalysis system; thus, harsher reaction conditions are generally required [35]. For this reason, coupling of aryl chlorides and aryl boronic acids is commonly selected as a standard reaction to demonstrate the high reactivity of a catalyst $[22,36]$. At a Pd loading of $0.1 \%$, 99\% yield of biphenyl was obtained after heating chlorobenzene and benzeneboronic acid at $80^{\circ} \mathrm{C}$ for $6 \mathrm{~h}$ (Table 2 , entry 12). Other aryl chlorides bearing electron-giving groups such as $-\mathrm{OMe}$ or $-\mathrm{CF}_{3}$ gave high quantitative yields within $6 \mathrm{~h}$. Notably, the electron deficient 1-chloro-4-nitrobenzene also gave a high and reproducible yield in 8 continuous cycles (Table 2, entry 14). More impressively, coupling of benzyl halides with phenylboronic acid, which is a rarely reported, challenging reaction but important in heterogeneous catalysis [22], also performed very well (Table 2, entry 15,16$)$. This further demonstrates the high activity of PPPd for coupling benzyl halide and phenylboronic acid.
Encouraged by these impressive results, we further exposed the substrate to vinyl and alkyl boronic acids. The alkylation of aryl halides with vinyl- or alkylboron derivatives may provide a simple and easy way to obtain the corresponding aryl-vinyl or aryl-alkyl derivatives. Normally, these reactions are performed using 9-BBN compounds as reactants, which are not air stable thus difficult to operate, and the processes are not economical [37]. In the last decade, several palladium catalysts associated with electro-rich phosphine ligands have been developed to achieve the coupling of alkylboronic esters or acids in homogeneous conditions. To the best of our knowledge, very few cases have been reported for these reactions in heterogeneous systems, which typically gave very low yields [38,39]. In our system, the coupling of 2-bromo-6methylpyridine with (E)-(4-methoxystyryl) boronic acid occurred smoothly, leading to a 95\% isolated yield in the coupled product. The coupling of aryl iodide and aryl bromide with $n$-butylboronic acid was also successfully achieved in a longer reaction time. Interestingly, the aryl bromide reacted faster than the aryl iodide, which suggests that in the case of cross-coupling of aryl iodide with alkyl boronic acid, oxidative addition may not be the rate-limiting step of the catalytic cycle. Such phenomenon was also observed in a homogeneous Suzuki cross-coupling system [40]. Importantly, such reactions cannot be achieved with homogeneous catalyst of $\mathrm{Pd}\left(\mathrm{PPh}_{3}\right)_{4}$.

The biaryl motif is of great importance in biological products, as well as in asymmetric catalysis, thus stimulating numerous construction methodologies [41]. In addition to the original and the most widely used copper-mediated Ullmann couplings, palladium-catalyzed reductive homo-couplings of aryl halides have also been reported, and advantages were found [42]. One big challenge in Pd mediated homo-coupling of aryl halides is 
the generation of $\mathrm{Pd}^{0}$ which always require inconvenient reaction conditions [41]. By supporting palladium NPs on a porous MOF, Jiang and co-workers reported a heterogeneous $\mathrm{Pd}^{\circ}$ Catalyst, $\mathrm{Pd} /$ MIL-101, which is efficient for Ullmann couplings and also found that $\mathrm{Pd}^{\mathrm{II}}$ is not active [36]. During our examination of the coupling of aryl iodide and butylboronic acid, a significant amount of homocoupling biaryl product was observed. Considering the mixedvalence nature of $\mathrm{Pd}^{0} \mathrm{Pd}^{\mathrm{II}}$ in $\mathrm{PPPd}$, the biaryl compound must come from the $\mathrm{Pd}^{\mathrm{II}}$-catalyzed Ullmann coupling reaction.

Table 3

Palladium-catalyzed reductive homo-coupling of aryl halides and oxidative homocoupling of aryl boronic acid ${ }^{[a]}$.

Entry

[a] Reaction conditions: Aryl halide $(0.5 \mathrm{mmol}), \mathrm{K}_{3} \mathrm{PO}_{4}(1.5 \mathrm{mmol}), \mathrm{H}_{2} \mathrm{O} / \mathrm{EtOH}$ $(2: 3 \mathrm{v} / \mathrm{v}, 2 \mathrm{ml}), \operatorname{PPPd}(0.2 \mathrm{~mol} \% \mathrm{Pd}), 80^{\circ} \mathrm{C}, 20 \mathrm{~h}$, under air.

[b] GC yield, isolated yield in parentheses.

[c] $0.2 \mathrm{~mol} \% \mathrm{Pd}\left(\mathrm{PPh}_{3}\right)_{4}$ was used instead of PPPd.

[d] $0.2 \mathrm{~mol} \% \mathrm{PdCl}_{2}$ was used instead of PPPd.

[e] Arylboronic acid $(0.5 \mathrm{mmol}), \mathrm{K}_{3} \mathrm{PO}_{4}(1.5 \mathrm{mmol}), \mathrm{H}_{2} \mathrm{O} /$ EtOH $(2: 3 \mathrm{v} / \mathrm{v}, 2 \mathrm{ml})$, PPPd

(0.1 mol\% Pd), $80^{\circ} \mathrm{C}, 6 \mathrm{~h}$, under air.
Further experiments quickly revealed that under the presence of PPPd, aryl iodide, bromide, and chloride were all homocoupled to the corresponding biaryl products in excellent yields (Table 3 , entry 1-3). In comparison with the highly active PPPd catalyst, $\mathrm{Pd}\left(\mathrm{PPh}_{3}\right)_{4}$ and $\mathrm{PdCl}_{2}$ were found not active under the same conditions (Table 3 , entry 3 ), indicating that the porous polymer supported mix-valence palladium was essential for the Ullmann coupling reaction. The oxidative homo-coupling of aryl boronic acids was also conducted successfully, leading to biaryl products under conditions similar to Suzuki coupling reactions but without halides and under an air atmosphere (Table 3, entry 4-5). Furthermore, the exceptionally high activity and selectivity for the biaryl synthesis in an aqueous solvent at a moderate temperature shows great potential for practical applications.

The direct conversion of $\mathrm{C}-\mathrm{H}$ bonds into $\mathrm{C}-\mathrm{O}, \mathrm{C}-$ halogen, $\mathrm{C}-\mathrm{C}$ bonds has attracted significant attention but remains a critical challenge in organic chemistry. Recently, Cohen et al. reported a thiocatechol-functionalized MOF supported recyclable Pd catalyst that can selectively catalyze chelate-directed functionalization of $\mathrm{C}-\mathrm{H}$ bonds [23]. However, the synthesis of this MOF substrate was costly and a high palladium loading (5-15 mol\%) was required to obtain good yields, which undoubtedly inhibits its practical applications. To the best of our knowledge, although numerous of porous polymer supported palladium catalysts have been reported in the literature, applications in direct $\mathrm{C}-\mathrm{H}$ bond functionalization are quite rare. The outstanding performance of PPPd in the reactions of $\mathrm{C}-\mathrm{C}$ bond formation motivated us to further investigate its catalytic activity in regioselective heterogeneous $\mathrm{C}-\mathrm{H}$ functionalization. To our delight, successful $\mathrm{C}-\mathrm{H}$ oxidation of benzoquinoline was achieved regioselectively to give benzo[h] quinolin-10-yl acetate in a quantitative yield with iodobenzene diacetate $\left[\mathrm{PhI}(\mathrm{OAc})_{2}\right]$ as the oxidant and $0.4 \mathrm{~mol} \%$ of the PPPd catalyst in hot $\mathrm{MeCN}$ within $12 \mathrm{~h}$ under an air atmosphere. When the solvent was changed to methanol $(\mathrm{MeOH})$ or 2,2,2-trifluoroethanol $\left(\mathrm{CF}_{3} \mathrm{CH}_{2} \mathrm{OH}\right)$, alkoxy functional groups of $-\mathrm{OMe}$ and $-\mathrm{OCH}_{2} \mathrm{CF}_{3}$ were successively and selectively added. When NXS was used as

Table 4

Palladium-catalyzed direct $\mathrm{C}-\mathrm{H}$ bond selective alkoxylation and halogenation reactions ${ }^{[a]}$.

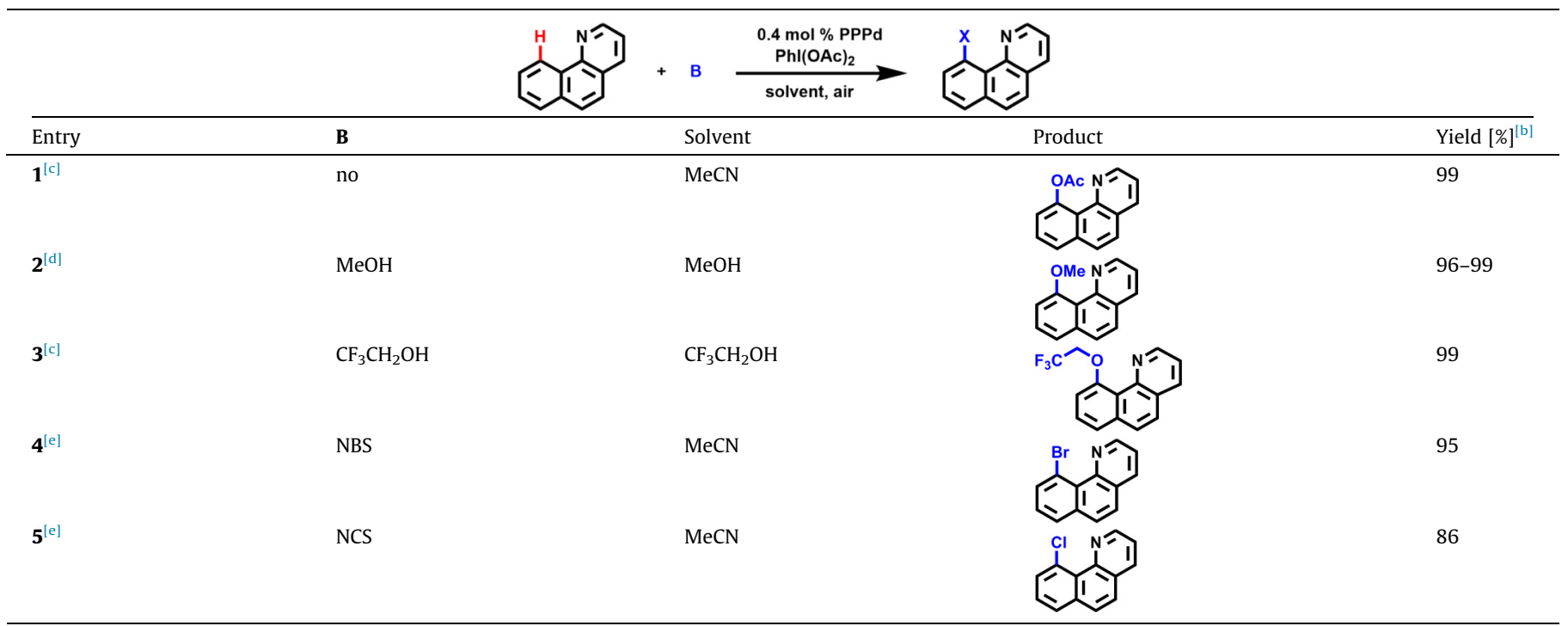

[a] Reaction conditions: benzoquinoline $(0.5 \mathrm{mmol}), \mathrm{PhI}(\mathrm{OAc})_{2}(1.0 \mathrm{mmol})$, solvent $(4 \mathrm{ml})$, PPPd $(0.4 \mathrm{~mol} \% \mathrm{Pd})$, under air.

[b] Determined by GC-MS.

[c] $100{ }^{\circ} \mathrm{C}, 12 \mathrm{~h}$.

[d] $70^{\circ} \mathrm{C}, 6 \mathrm{~h}, 5$ cycles.

[e] $\mathrm{No} \mathrm{PhI}(\mathrm{OAc})_{2}$ was used. NXS (1.25 mmol), $85^{\circ} \mathrm{C}, 20 \mathrm{~h}$. Multi-halogenation was observed when more NXS was applied. 
Table 5

Palladium-catalyzed intermolecular regioselective $\mathrm{C}-\mathrm{H}$ alkenylation of indole ${ }^{[\mathrm{a}]}$.

\begin{tabular}{|c|c|c|c|c|c|}
\hline Entry & Catalyst & $\mathrm{Pd}(\mathrm{mol} \%)$ & Time (h) & Yield (\%) & Ref. \\
\hline 1 & $\mathrm{Pd}(\mathrm{OAc})_{2}$ & 10 & 18 & 66 & {$[24]$} \\
\hline 2 & PPPd & 0.2 & 12 & 88-92 (5 cycles) & this work \\
\hline
\end{tabular}

[a] Reaction conditions: Indole $(0.5 \mathrm{mmol}), \mathrm{Cu}(\mathrm{OAc})_{2}(0.8 \mathrm{mmol})$, alkene $(1.5 \mathrm{mmol})$, DMF/DMSO $(9: 1 \mathrm{v} / \mathrm{v}, 4 \mathrm{ml})$, air. Yield determined by GC.

the oxidant in replacement of $\mathrm{PhI}(\mathrm{OAc})_{2}$, good to excellent yields of halogenation products were obtained (Table 4, entries 4 and 5). Though only $0.4 \mathrm{~mol} \%$ catalyst was used, PPPd-catalyzed $\mathrm{C}-\mathrm{H}$ oxidation of benzoquinoline showed higher activity than the reported $\mathrm{Pd}(\mathrm{OAc})_{2}$ homogeneous catalysts [43] and much higher activity than the Pd/MOF heterogeneous catalysts [23]. The higher reactivity of PPPd can be rationalized in the following manner. First, the spatial-isolated palladium atoms in the porous network with high surface area provide more active sites. Second, the fully organic nature of the support with good solvent wettability can accelerate the mass diffusion of organic reactant molecules thus promoting the catalytic cycle. Third, phosphine oxide inside the framework coordinates with palladium to keep the resting state of Pd in a high active form [44]. Fourth, although the $\mathrm{Pd}^{\mathrm{II} / 0}$ or $\mathrm{Pd}^{\mathrm{II} / \mathrm{IV}}$ cycle process is still a debated topic in C-H activation [45], PPPd with both $\mathrm{Pd}^{0}$ and $\mathrm{Pd}^{\mathrm{II}}$ can possibly serve both paths, thus enhancing the overall conversion rate.

Intermolecular regioselective $\mathrm{C}-\mathrm{H}$ alkenylation of $\mathrm{N}$-free indole was also achieved with good yield and selectivity (Table 5), under similar reaction conditions as $\mathrm{Pd}(\mathrm{OAc})_{2}$-catalyzed homogeneous systems, but with much lower Pd loading and shorter reaction time.[24] As shown in table 5, 90\% yields of C-3 alkenylation product was obtained with only $0.2 \mathrm{~mol} \%$ PPPd loading in $12 \mathrm{~h}$, which is two magnitudes more efficient than the reported $\mathrm{Pd}(\mathrm{OAc})_{2}$ system. Moreover, the PPPd catalyst can be easily recovered and reused without significant loss of activity after five runs.

\section{Conclusions}

Overall, we presented a convenient one-step cross knitting strategy to immobilize and stabilize the unstable organometallic homogeneous catalyst $\mathrm{Pd}\left(\mathrm{PPh}_{3}\right)_{4}$ inside a porous flower-like 3D organic microporous polymer. The initially coordinated Pd as well as the fast forming microporous structure ensured the sitedispersion of Pd. Electro-beam irradiation induced metal atoms aggregation-crystallization was observed directly under HRTEM imaging process for the first time on POPs. The PPPd introduced here is easy to synthesize, simple in operation, low in cost, and stable in air and moisture. Moreover, it exhibits excellence in activity and selectivity towards various challenging heterogeneous catalytic systems, including $\mathrm{C}-\mathrm{C}$ bond formation reactions in challenging Suzuki-Miyaura cross-couplings of aryl chlorides and alkyl boronic acids, reductive and oxidative biaryl synthesis in aqueous medium, and $\mathrm{C}-\mathrm{H}$ regioselective activation/functionalization reactions. The catalyst can be easily recovered, with no detectable metal leakage and no decrease in activity, revealing its great potential in practical applications. The strategy developed here provides practical method for easy-to-make yet highly efficient heterogeneous catalysis. Application of such a strategy to other organometallics such as $\mathrm{Ni}\left(\mathrm{PPh}_{3}\right)_{4}, \mathrm{RuCl}_{2}\left(\mathrm{PPh}_{3}\right)_{4}$ is currently on going in our lab.

\section{Acknowledgement}

Financial support was provided by KAUST competitive grant URF/1/1723, Saudi Arabia.

\section{Appendix A. Supplementary material}

Supplementary data associated with this article can be found, in the online version, at https://doi.org/10.1016/j.jcat.2017.08.030.

\section{References}

[1] N. Mizuno, M. Misono, Heterogeneous catalysis, Chem. Rev. 98 (1998) 199218.

[2] D. Astruc, F. Lu, J.R. Aranzaes, Nanoparticles as recyclable catalysts: the frontier between homogeneous and heterogeneous catalysis, Angew. Chem. Int. Ed. 44 (2005) 7852-7872.

[3] C. Coperet, A. Comas-Vives, M.P. Conley, D.P. Estes, A. Fedorov, V. Mougel, H. Nagae, F. Núñez-Zarur, P.A. Zhizhko, Surface organometallic and coordination chemistry toward single-site heterogeneous catalysts: strategies, methods, structures, and activities, Chem. Rev. 116 (2016) 323-421.

[4] C. Copéret, M. Chabanas, R. Petroff Saint-Arroman, J.M. Basset, Homogeneous and heterogeneous catalysis: bridging the gap through surface organometallic chemistry, Angew. Chem. Int. Ed. 42 (2003) 156-181.

[5] L. Pan, H. Liu, X. Lei, X. Huang, D.H. Olson, N.J. Turro, J. Li, RPM-1: A Recyclable nanoporous material suitable for ship-in-bottle synthesis and large hydrocarbon sorption, Angew. Chem. Int. Ed. 42 (2003) 542-546.

[6] X. Wang, L. Xie, K.-W. Huang, Z. Lai, A rationally designed amino-borane complex in a metal organic framework: a novel reusable hydrogen storage and size-selective reduction material, Chem. Commun. 51 (2015) 7610-7613.

[7] A. Dhakshinamoorthy, H. Garcia, Catalysis by metal nanoparticles embedded on metal-organic frameworks, Chem. Soc. Rev. 41 (2012) 5262-5284.

[8] J. Liu, L. Chen, H. Cui, J. Zhang, L. Zhang, C.-Y. Su, Applications of metal-organic frameworks in heterogeneous supramolecular catalysis, Chem. Soc. Rev. 43 (2014) 6011-6061.

[9] H. Yan, H. Cheng, H. Yi, Y. Lin, T. Yao, C. Wang, J. Li, S. Wei, J. Lu, Single-atom Pd1/graphene catalyst achieved by atomic layer deposition: remarkable performance in selective hydrogenation of 1, 3-butadiene, J. Am. Chem. Soc. 137 (2015) 10484-10487.

[10] B. Qiao, A. Wang, X. Yang, L.F. Allard, Z. Jiang, Y. Cui, J. Liu, J. Li, T. Zhang, Singleatom catalysis of CO oxidation using Pt1/FeOx, Nat. Chem. 3 (2011) 634-641.

[11] X.-F. Yang, A. Wang, B. Qiao, J. Li, J. Liu, T. Zhang, Single-atom catalysts: a new Frontier in heterogeneous catalysis, Acc. Chem. Res. 46 (2013) 1740-1748.

[12] M. Dhiman, B. Chalke, V. Polshettiwar, Organosilane oxidation with a half million turnover number using fibrous nanosilica supported ultrasmall nanoparticles and pseudo-single atoms of gold, J. Mater. Chem. A 5 (2017) $1935-1940$.

[13] P. Gautam, M. Dhiman, V. Polshettiwar, B.M. Bhanage, KCC-1 supported palladium nanoparticles as an efficient and sustainable nanocatalyst for carbonylative Suzuki-Miyaura cross-coupling, Green Chem. 18 (2016) 58905899.

[14] M. Dhiman, V. Polshettiwar, Ultrasmall nanoparticles and pseudo-single atoms of platinum supported on fibrous nanosilica (KCC-1/Pt): engineering selectivity of hydrogenation reactions, J. Mater. Chem. A 4 (2016) 1241612424.

[15] G. Vilé, D. Albani, M. Nachtegaal, Z. Chen, D. Dontsova, M. Antonietti, N. López, J. Pérez-Ramírez, A Stable Single-Site Palladium Catalyst for Hydrogenations, Angew. Chem. Int. Ed. 54 (2015) 11265-11269.

[16] L. Tan, B. Tan, Hypercrosslinked porous polymer materials: design, synthesis, and applications, Chem. Soc. Rev. (2017), https://doi.org/10.1039/ c1036cs00851h.

[17] Q. Sun, Z. Dai, X. Meng, L. Wang, F.-S. Xiao, Task-specific design of porous polymer heterogeneous catalysts beyond homogeneous counterparts, ACS Catal. 5 (2015) 4556-4567. 
[18] X. Wang, Y. Liu, X. Ma, S.K. Das, M. Ostwal, I. Gadwal, K. Yao, X. Dong, Y. Han, I. Pinnau, Soluble polymers with intrinsic porosity for flue gas purification and natural gas upgrading, Adv. Mater. 29 (2017).

[19] P. Kaur, J.T. Hupp, S.T. Nguyen, Porous organic polymers in catalysis: opportunities and challenges, ACS Catal. 1 (2011) 819-835.

[20] J.-K. Sun, W.-W. Zhan, T. Akita, Q. Xu, Toward homogenization of heterogeneous metal nanoparticle catalysts with enhanced catalytic performance: soluble porous organic cage as a stabilizer and homogenizer, J. Am. Chem. Soc. 137 (2015) 7063-7066.

[21] B. Li, R. Gong, W. Wang, X. Huang, W. Zhang, H. Li, C. Hu, B. Tan, A new strategy to microporous polymers: knitting rigid aromatic building blocks by external cross-linker, Macromolecules 44 (2011) 2410-2414.

[22] B. Li, Z. Guan, W. Wang, X. Yang, J. Hu, B. Tan, T. Li, Highly dispersed Pd catalyst locked in knitting aryl network polymers for Suzuki-Miyaura coupling reactions of aryl chlorides in aqueous media, Adv. Mater. 24 (2012) 33903395.

[23] H. Fei, S.M. Cohen, Metalation of a thiocatechol-functionalized $\operatorname{Zr}($ IV)-based metal-organic framework for selective C-H functionalization, J. Am. Chem. Soc. 137 (2015) 2191-2194.

[24] N.P. Grimster, C. Gauntlett, C.R. Godfrey, M.J. Gaunt, Palladium-catalyzed intermolecular alkenylation of indoles by solvent-controlled regioselective CH functionalization, Angew. Chem. 117 (2005) 3185-3189.

[25] A. Mondal, N.R. Jana, Effect of size and oxidation state of platinum nanoparticles on the electrocatalytic performance of graphene-nanoparticle composites, RSC Adv. 5 (2015) 85196-85201.

[26] A.F. Heyduk, D.G. Nocera, Hydrogen produced from hydrohalic acid solutions by a two-electron mixed-valence photocatalyst, Science 293 (2001) 16391641.

[27] W. Chen, Z. Fan, Z. Lai, Synthesis of core-shell heterostructured $\mathrm{Cu} / \mathrm{Cu}_{2} \mathrm{O}$ nanowires monitored by in situ XRD as efficient visible-light photocatalysts, J. Mater. Chem. A 1 (2013) 13862-13868.

[28] M.M. Najafpour, Mixed-valence manganese calcium oxides as efficient catalysts for water oxidation, Dalton Trans. 40 (2011) 3793-3795.

[29] C.-C. Chang, A.R. Teixeira, C. Li, P.J. Dauenhauer, W. Fan, Enhanced molecular transport in hierarchical silicalite-1, Langmuir 29 (2013) 13943-13950.

[30] S. Hou, Y. Zou, X. Liu, X. Yu, B. Liu, X. Sun, Y. Xing, $\mathrm{CaF}_{2}$ and $\mathrm{CaF}_{2}: \operatorname{Ln}^{3+}(\mathrm{Ln}=\mathrm{Er}$ $\mathrm{Nd}, \mathrm{Yb}$ ) hierarchical nanoflowers: hydrothermal synthesis and luminescent properties, CrystEngComm 13 (2011) 835-840.

[31] M. Rimoldi, A. Nakamura, N.A. Vermeulen, J.J. Henkelis, A.K. Blackburn, J.T. Hupp, J.F. Stoddart, O.K. Farha, A metal-organic framework immobilised iridium pincer complex, Chemical Science 7 (2016) 4980-4984.
[32] S.A. Burgess, A. Kassie, S.A. Baranowski, K.J. Fritzsching, K. Schmidt-Rohr, C.M. Brown, C.R. Wade, Improved catalytic activity and stability of a palladium pincer complex by incorporation into a metal-organic framework, Journal of the American Chemical Society 138 (2016) 1780-1783.

[33] K. Justin Thomas, J.T. Lin, M. Velusamy, Y.T. Tao, C.H. Chuen, Color tuning in Benzo [1,2,5] thiadiazole-based small molecules by amino conjugation/ deconjugation: bright red-light-emitting diodes, Adv. Funct. Mater. 14 (2004) 83-90.

[34] M. Wang, X. Hu, P. Liu, W. Li, X. Gong, F. Huang, Y. Cao, Donor-acceptor conjugated polymer based on naphtho [1,2-c: $5,6-c]$ bis $[1,2,5]$ thiadiazole for high-performance polymer solar cells, J. Am. Chem. Soc. 133 (2011) 96389641.

[35] L. Yin, J. Liebscher, Carbon-carbon coupling reactions catalyzed by heterogeneous palladium catalysts, Chem. Rev. 107 (2007) 133-173.

[36] B. Yuan, Y. Pan, Y. Li, B. Yin, H. Jiang, A highly active heterogeneous palladium catalyst for the Suzuki-Miyaura and Ullmann coupling reactions of aryl chlorides in aqueous media, Angew. Chem. Int. Ed. 49 (2010) 4054-4058.

[37] H. Doucet, Suzuki-Miyaura cross-coupling reactions of alkylboronic acid derivatives or alkyltrifluoroborates with aryl, alkenyl or alkyl halides and triflates, Eur. J. Org. Chem. 2008 (2008) 2013-2030.

[38] A. Cwik, Z. Hell, F. Figueras, Suzuki-Miyaura cross-coupling reaction catalyzed by Pd/MgLa mixed oxide, Org. Biomol. Chem. 3 (2005) 4307-4309.

[39] G.W. Kabalka, L. Wang, R.M. Pagni, C.M. Hair, V. Namboodiri, Solventless Suzuki coupling reactions on palladium-doped potassium fluoride alumina, Synthesis (2003) 217-222.

[40] A.F. Littke, C. Dai, G.C. Fu, Versatile catalysts for the Suzuki cross-coupling of arylboronic acids with aryl and vinyl halides and triflates under mild conditions, J. Am. Chem. Soc. 122 (2000) 4020-4028.

[41] D.D. Hennings, T. Iwama, V.H. Rawal, Palladium-catalyzed (Ullmann-Type) homocoupling of aryl halides: a convenient and general synthesis of symmetrical biaryls via inter-and intramolecular coupling reactions, Org. Lett. 1 (1999) 1205-1208.

[42] S. Venkatraman, C.-J. Li, Carbon-carbon bond formation via palladiumcatalyzed reductive coupling in air, Org. Lett. 1 (1999) 1133-1135.

[43] L.V. Desai, K.L. Hull, M.S. Sanford, Palladium-catalyzed oxygenation of unactivated sp3 CH bonds, J. Am. Chem. Soc. 126 (2004) 9542-9543.

[44] A.K. Cook, M.S. Sanford, Mechanism of the palladium-catalyzed arene C-H acetoxylation: a comparison of catalysts and ligand effects, J. Am. Chem. Soc. 137 (2015) 3109-3118.

[45] T.W. Lyons, M.S. Sanford, Palladium-catalyzed ligand-directed C-H functionalization reactions, Chem. Rev. 110 (2010) 1147-1169. 